CARNETS DE Carnets de géographes

GÉOGRAPHES.

14 | 2020

Varia

\title{
Géographie et spiritualité
}

Quelque chose de $(\mathrm{du})$ « spirituel » en géographie

\section{Louisa Even}

\section{(2) OpenEdition}

Journals

Édition électronique

URL : https://journals.openedition.org/cdg/6132

DOI : $10.4000 /$ cdg. 6132

ISSN : 2107-7266

Éditeur

UMR 245 - CESSMA

Référence électronique

Louisa Even, « Géographie et spiritualité », Carnets de géographes [En ligne], 14 | 2020, mis en ligne le 30 décembre 2020, consulté le 21 mai 2021. URL : http://journals.openedition.org/cdg/6132 ; DOI : https://doi.org/10.4000/cdg.6132

Ce document a été généré automatiquement le 21 mai 2021.

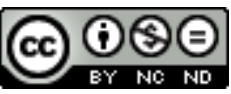

La revue Carnets de géographes est mise à disposition selon les termes de la Licence Creative Commons Attribution - Pas d'Utilisation Commerciale - Pas de Modification 4.0 International. 


\title{
Géographie et spiritualité
}

\author{
Quelque chose de $(\mathrm{du})$ «spirituel » en géographie
}

\section{Louisa Even}

«Le spirituel », au sens le plus ordinaire de la langue française ${ }^{1}$, peut-il être une notion utilisée dans les divers champs de savoir des sciences humaines et sociales et notamment en géographie? Comment le chercheur peut intégrer «le spirituel» dans son analyse, et comment ce dernier peut-il être considéré comme un paradigme de la géographie? C'est un intérêt pour ces questions, dont les réponses ne sont pas évidentes à priori, car le sujet est complexe, qui a constitué le point de départ de notre questionnement ${ }^{2}$. Au seuil de cette contribution, il paraît important de préciser que le sens que nous réservons à «spirituel » (et spiritualité) et dans lequel s'inscrit cette réflexion, est très large, ainsi :

«Le mot spiritualitas que l'on rencontre parfois dans les textes philosophiques à partir du $12^{\text {ème }}$ siècle n'a pas de contenu spécifiquement religieux : il désigne la qualité de ce qui est spirituel, c'est-à-dire indépendant de la matière. En fait, la spiritualité est un concept moderne, utilisé seulement depuis le 19ème siècle. » (Vauchez, 1994).

2 Par ailleurs, si nous postulons que l'être humain est, du fait de sa nature humaine même, un être (bicéphale) corporel ET «spirituel», il est incontournable de reconnaître aujourd'hui à la catégorie du « spirituel » une place aussi déterminante que l'étude des matérialités dans nos recherches :

"Sous peine d'obsolescence et de délégitimation, les géographes ont eu à trouver des approches autres que celle qui consiste à produire un discours sur le monde matériel, le monde réel, et à ignorer superbement le besoin de lui accorder un statut ontologique. En cela, les géographes sont sur la voie de renouer explicitement, sinon avec la philosophie, du moins avec une approche qui ne soit plus exclusivement réaliste. » (Chamussy, 2003, p. 64).

Dès lors, on voit bien que la question de savoir si « le spirituel » peut être considéré comme un concept scientifique opératoire intéresse toutes les sciences humaines et sociales, alors pourquoi cette méfiance, voire ce complexe vis-à-vis du « spirituel »? Il nous semble pourtant qu'il ne peut, au sens où nous l'entendons, que contribuer à l'enrichissement des problématiques sur l'humain. Ainsi, «le spirituel » ne devrait pas être écartée mais au contraire être utilisé comme grille de lecture dans la connaissance : 
"Si de nos jours, on entend par spiritualité une sorte de savoir supérieur, dispensé par des experts et par des maîtres spirituels, on en fait quelque chose d'abstrait, d'austère, et on la réduit à une discipline intellectuelle. Mieux vaudrait dire "le spirituel», comme on dit «le divin », en ouvrant un champ non seulement de réflexion, mais d'expérience » (Kelen, 2015, p35).

4 Par conséquent, et comme prolongement à ces nombreuses interrogations et débats qui ont cours depuis plusieurs années maintenant, et dont nous n'aborderons que certains aspects, l'article s'attache à montrer que la notion de «spirituel » (qui a régulièrement été convoquée dans les travaux de géographie humaine et sociale (Dejean, 2013), soit implicitement, soit explicitement ${ }^{3}$ ) peut notamment s'illustrer dans les liens environnement/spirituel-spiritualité ou nature/spirituel-spiritualité (et peuvent d'ailleurs trouver des prolongements dans le fait religieux). Nous avons choisi de montrer, en premier lieu, que l'usage du «spirituel» en tant que tel est à la fois informulé (mais ostensible) dans de nombreux travaux sur les représentations territoriales, les images mentales des lieux et autres recherches sur les perceptions spatiales et les comportements humains; et à la fois sous-entendu (mais omniprésent) et convoqué en géographie dans les dialectiques visible/invisible ${ }^{4}$, matériel/immatériel, corporel/idéel par exemple. En second lieu, nous verrons que c'est sans doute dans les travaux concernant l'environnement (et sa protection) que l'opposition explicite matériel/spirituel est la plus saillante et que, de ce fait, les positionnements scientifiques laissent apparaître-là le plus clairement (y compris dans la langue) la catégorie du «spirituel» ou la spiritualité; cette dernière devenant dès lors intimement associée au genre humain et à son action. Les liens environnement/ spiritualité sont notamment manifestés dans le concept de développement durable, d'où naît une intelligence de la nature ${ }^{5}$ et de l'être humain intégral.

5 En géographie, il n'est guère d'usage d'utiliser le terme de « spirituel » en tant que tel, et pourtant, du fait de l'articulation classique homme/espace ou social/spatial dans l'analyse, on est obligé de reconnaître que les causalités ne peuvent pas se résumer à de pures matérialités :

"L'espace géographique qui accueille la vie sociale et au sein duquel s'accomplissent les destinées humaines, n'a pas cette objectivité placide que la géographie classique et ses versions positivistes retenaient naguère implicitement. (...) L'homme qui se représente l'espace et son milieu, qui le façonne et le transforme est à la fois une conscience, une psyché, un sujet philosophique et politique, un individu, une personne, un être humain et un être social» (Di Méo, 1999, p. 56).

6 Par la voix de Guy Di Méo nous voyons ici que les aspects relevant du « spirituel » et de toute disposition humaine intérieure («Conscience», "Psyché» nous dit-il) peuvent intervenir comme facteur explicatif de n'importe quelles pratiques sociales et spatiales. Certains géographes nous ont même parlé (et ce n'est pas qu'une métaphore) de paysages intérieurs :

«Le paysage n'existe pas en soi, il est un regard particulier porté sur un fragment de la réalité géographique, une "invention" historique et culturelle. (...) On a ainsi parlé de paysage social, de paysage statistique, législatif, politique, audiovisuel, pour ne rien dire des paysages intérieurs » (Pinchemel, 1997, p. 376).

7 Mais ces propos laissent également supposer que quels que soient les objets géographiques étudiés, déborder de l'objectivité ${ }^{6}$ de la réalité humaine visible et palpable, fait surgir d'éventuels doutes scientifiques (Besse, 2004, p. 1-5). En effet, dès lors qu'il s'agit d'étudier des représentations mentales et des perceptions de l'espace (Plouchart, 1997), des images mentales des lieux ou l'imaginaire des espaces (Chivallon, 
2008), voire l'esprit des lieux (Lévy, Lussault, 2000), des précautions scientifiques spécifiques sont prises car cela pose au chercheur des questions méthodologiques sérieuses (Debarbieux, 2004, non paginé); Antoine Bailly pose lui aussi les questions importantes de limites scientifiques et de méthodologie à préciser :

"L'humanisme est ainsi irruption du monde poétique dans le monde scientifique et prise de conscience de l'explicitation nécessaire de sa propre subjectivité (...). D'où l'indispensable appel à de nouveaux moyens pour la recherche géographique, des textes littéraires aux analyses psychologiques, à condition de préserver un esprit de rigueur dans lequel le choix des méthodes est clairement lié à celui des objectifs » (Bailly, 1990, p. 212-219).

8 En géographie humaine, et suivant cette dialectique homme/espace, les rapports sociaux sont envisagés du point de vue de leur influence sur les rapports spatiaux (ségrégation, mobilité, comportements...). Quelles que soient les combinaisons géographiques qui en découlent, dans tous les cas l'hypothèse d'un effet de lieu est à la base du discours. Les géographes tentent finalement de montrer comment s'exprime l'interférence du social et du spatial dans les pratiques particulières à partir des représentations que les individus (et les groupes sociaux) ont de leurs espaces de vie aspects subjectifs et idéels- (et non plus seulement dans la disposition des unités de production et d'habitat ${ }^{7}$-aspects objectifs et matériels-) :

«Le territoire serait donc un édifice conceptuel reposant sur deux piliers complémentaires, souvent présentés comme antagonistes en géographie: le matériel et l'idéel. (...) Appropriation et enracinement se manifestent par des éléments matériels mais aussi idéels » (Elissalde, 2004, non paginé).

9 Quoiqu'il en soit, dans cette géographie humaniste, il est prôné un recours plus systématique à la perception sensible et sensitive. Il s'agit de comprendre l'expérience que les individus font de l'espace: non plus simplement expérience physique mais expérience mentale, et partant, spirituelle aussi. Ainsi, saisir et expliquer quel sens ils donnent aux lieux constitue un objet d'étude phénoménologique à part entière (le sens des lieux, sense of place (Gould, White, 1984)) :

"La géographie phénoménologique, des "espaces vécus", des perceptions et de leurs représentations est basée sur le principe de la relativité du savoir et aussi sur l'étude du comportement des individus, ainsi, la géographie de la perception permet-elle de comprendre les schémas de comportement, mais elle dégage ce que les anglophones appellent le "sense of place", c'est-à-dire le sens de l'espace territorial » (Bailly, 1977, p. 83-95).

10 Ce sens des lieux et cette perception des lieux sont appréhendés par des outils d'analyse tels que les cartes mentales, les enquêtes directes, les parcours commentés et toutes données collectées empiriquement. En réalité, cette géographie est très ambitieuse puisqu'elle propose d'objectiver le subjectif et le sensible, propres à chacun :

«Les savoirs géographiques vernaculaires sont un engagement de tous les sens, ce qui les fonde dans une expérience ontologique de l'espace et du milieu. Il en résulte qu'ils sont nécessairement subjectifs et contextualisés. De sorte qu'ils ne peuvent être que pluriels. Si l'on peut repérer des caractères communs à tout savoir vernaculaire, les structures et les contenus sont toujours différents, comme les cultures mais aussi les individus dont ils sont l'expression. »(Colignon, 2004, non paginé).

11 Ces propos confirment bien qu'il s'agit d'une approche qui invite le subjectif et le singulier, voire "le spirituel » puisque l'analyse géographique convoque l'expérience ontologique nous dit l'auteur (rejoignant par-là la pensée de Henri Chamussy cité au début de cet article) : 
«(...) L'objectif fondamental demeure la construction du sens de l'espace. Car la géographie trouve là sa justification de science sociale et devient pertinente et passionnante (...). » (André, 1998, p. 90).

12 Or on sait que l'anthropologie a mis au jour que tous les êtres humains n'ont pas la même compréhension du monde ni de ce que signifie être au monde; parmi ces ontologies, aucune ne surclasse les autres (Descola, Ingold, Lussault, 2014). Existe-t-il alors un point de vue neutre à partir duquel les étudier et les comparer ? Par ailleurs, la démarche se fonde sur la dimension biographique et existentielle :

"En Géographie, l'attitude phénoménologique se marque surtout par un intérêt de connaissance des intentions des acteurs, de leurs rapports aux lieux, de leur "espace vécu", de leurs représentations de l'espace; voire par la compréhension des logiques sociales qui sont associées à des formes spatiales autrement incompréhensibles ou qui seraient "tombées du ciel". Elle a donné du sens à la contestation de la Géographie asociale et apolitique des "quantitativistes" anglophones inspirés par les modèles de l'économie spatiale et devrait permettre en principe d'éviter ainsi le spatialisme » (Brunet, 1995, p. 382).

13 Roger Brunet montre également que cette géographie interroge ce qui donne sens à l'existence et aux lieux de l'existence et qui ne saurait se réduire aux composantes matérielles et quantifiables dans l'espace ; idem chez Jean-Pierre Paulet pour qui :

"Comprendre le sens et la valeur que l'on accorde aux lieux a, en définitive, pour objet de faire progresser le bien-être » (Paulet, 2002, p. 4).

Cette géographie a été beaucoup critiquée, notamment dans ses méthodes et du fait de sa subjectivité, car c'est la représentation qui est le cœur de la démonstration et non plus les éléments visibles. Pourtant les résultats de telles recherches permettent de mettre en évidence les éventuels décalages et/ou convergences entre éléments matériels et représentations sociales et culturelles des matérialités (Plouchart, 1999). Ces recherches ont vocation à souligner des disparités spatiales, des inégalités territoriales, au minimum des différenciations dans l'espace d'un comportement étudié (Di Méo, 1991). Mais c'est toujours la dimension humaine et sensible qui est privilégiée, pour faire émerger une géographie des espaces vécus, perçus et représentés (Frémont, 1976). Par ailleurs, rejoignant là Armand Frémont, Antoine Bailly (pour qui « la région, la ville, le quartier miroirs de nos Sociétés sont à la fois rêves et cauchemars " (Bailly, 1991, p. 161-167)) propose une "géographie du bien-être " ou de "la satisfaction territoriale ", dans laquelle il déploie lui aussi une analyse usant d'éléments vécus, perçus ou représentés. On reproche à cette géographie d'être trop descriptive, subjective, idéelle et de surévaluer le rôle de l'individu et de la liberté individuelle : mais comment inclure « le spirituel » dans l'analyse autrement qu'à ce prix ? Par ailleurs, il semble bien que ce type de géographie des territoires prend le mieux en compte toute la complexité de l'être humain, qui, par ses pratiques et par son appropriation de l'espace, est vu selon Barel comme un animal territorialisateur : "Ainsi l'homme, parce qu'il est un animal social, est aussi un territorialisateur» (Barel, 1986). En effet, nous saisissons les territoires à travers nos représentations c'est-à-dire les images mentales qui émergent de notre personnalité ou de notre culture, de notre désir de comprendre, de notre volonté d'agir. Nous voyons les territoires à travers ce que nous sommes et notre façon de penser, à travers ce que nous voulons. Ainsi, les représentations des territoires sont multiples comme sont multiples les hommes qui les pensent (Bailly, Baumont, Huriot, Sallez, 1995). Cette entrée thématique par le territoire approprié, à dimension affective, subjective et symbolique se retrouve souvent dans les travaux géographiques sur les morphologies urbaines, et notamment les quartiers de grands ensembles de logements sociaux (Plouchart, 1999). Dans ces ZUP en effet, tous les arts de rue d'une manière 
générale, mais surtout le rap ou le slam français, ont souvent célébré cette appartenance territoriale très forte dans les cités :

«(...) J'connais bien ses rouages, j'connais bien ses virages, y'a tout l'temps du passage, y'a plein d'enfants pas sages, j'veux écrire une belle page, ville aux cent mille visages, St-Deniscentre mon village. (...)» (Grand Corps Malade, 2006).

La pratique spécifie en quelque sorte le territoire et en détermine des contenus, des limites et des frontières (Lynch, 1999), mais en produit également de l'attachement et de l'affectivité :

« Nous pouvons constater de manière transversale que l'attachement au lieu est envisagé dans une perspective phénoménologique, c'est-à-dire qu'il est relatif à l'expérience vécue des individus. En effet, l'attachement au lieu se développe lorsque l'environnement est considéré par l'individu comme un espace qui a du sens, avec lequel il se familiarise par son expérience » (Caro, 2019).

Enfin, les perceptions et comportements territoriaux, et notamment cet attachement à son territoire de vie (son environnement) ont également été rapprochés des écologies animales. Cette lecture particulière donne lieu à des modélisations spatiales que l'écologue urbain construit sur le postulat que le cadre de vie (l'environnement) conditionne non seulement les représentations mais aussi les comportements des individus (genres de vie). Par conséquent, la géographie des perceptions de l'environnement peut être mobilisée pour traiter et interpréter toute pratique, tout comportement, tout rapport, toute utilisation de l'espace et tout attachement aux territoires (Feildel, 2010). Elle a recours aux ambiances et aux émotions ${ }^{8}$; les expériences vécues, ambiances perçues ${ }^{9}$ ou représentées rendent compte de la relativité, de la subjectivité et de la symbolique de l'environnement appréhendé par l'individu, et relèvent des sphères affectives autant que spirituelles.

Comment « le spirituel» (ou la spiritualité) est explicatif dans le lien homme-milieu? Comment entre-t-il dans le processus de transformation (et d'usage) des paysages? Quelle est la place de ce rapport social particulier dans l'aujourd'hui de notre relation à la nature (protection/préservation) ? Voilà au préalable quelques-unes des questions qui se posent et qui invitent à définir la notion d'environnement. En effet, on est obligé de reconnaître qu'en dépit des efforts de définition et de clarification du concept ${ }^{10}$, il subsiste encore beaucoup d'ambiguïté aujourd'hui dans cette notion. Elle désigne, au plus général en géographie, la relation homme-milieu. Ainsi, la notion d'environnement pour Gabriel Rougerie (Rougerie, 1975) privilégie le cadre physique (naturel) ce qui donne plus d'importance à la notion de milieu transformé par l'homme, acteur. La distinction se fait ici entre les cadres de vie où l'homme est dominé par son milieu (" environnement ou cadres de vie écologiques»), et ceux où il domine son milieu ("environnement ou cadres de vie éthologiques»). Retenons donc que, d'une part, l'environnement, dans lequel l'homme est "facteur géographique " repose sur une représentation subjective du monde réel, et Pierre Gentelle de nous rappeler : «C'est qu'en effet, l'environnement est un donné, vécu et perçu par les individus " (Gentelle, 2008, p20-21); et que, d'autre part, la nature de la représentation (et l'imaginaire) conditionne le bien-être (et même le "bonheur d'habiter" des individus (Plouchart, 1997)). Pour mettre au jour une telle géographie du "bonheur d'habiter» (ou au contraire du mal-vivre, des pathologies socio-spatiales), il reste donc essentiel de considérer les images mentales des lieux, et de comprendre comment ces images frappent les esprits au point d'influencer les décisions des individus et d'en conditionner leur interprétation du monde; c'est précisément à ce titre, que la 
dimension spirituelle est très présente, bien que non formulée. Par ailleurs, cette notion de «spirituel» suppose une interdisciplinarité de fait dans la démarche; et Christine Baron d'affirmer que :

«Le monde a cessé d'être un "en soi» dont la science devrait offrir une interprétation correcte et unique. Le monde est devenu précisément "ce dont on a une conception", et ce, particulièrement dans les disciplines auxquelles nous nous intéressons dans ce cadre. L'homme n'est pas dans un rapport passif à l'espace mais il construit (ou éventuellement il détruit!) les espaces qu'il habite et en forge des représentations variées; ainsi les représentations de la philosophie, de l'histoire, de la sociologie et de la littérature sont-elles annexées à la réflexion géographique comme autant de manières de s'approprier un environnement. (...) Faisant droit à la singularité et reconnaissant une pertinence aux représentations imaginaires que chacun se forge de son propre espace, parmi lesquels la littérature et les arts ont un rôle privilégié, cette géographie suppose non seulement une démarche plus globale et une anthropologie du rapport au lieu, mais elle implique que le lieu soit défini par les usages qu'il suppose (lieux de mémoire par exemple)» (Baron, 2011, p. 42-50).

18 Cet exemple des lieux de mémoire, cité par l'auteur, renvoie bien à des formes ou d'autres de «spirituel » ou de spiritualités dans les lieux. De même, la mémoire des lieux (Chevalier, Lefort, 2016) montre que l'environnement (au sens large de «l'espace géographique ») se transforme en signe personnel, en identité propre, en patrimoine hérité et en souvenirs dont on fait mémoire, et qui renvoient bien à des formes du «spirituel». La notion de «spirituel» est également présente dans ce qu'on appelle communément l'esprit des lieux, là les mots parlent d'eux-mêmes, et ouvre tout un imaginaire :

«La géographie imaginaire aborde bien «ce processus de recomposition, de recréation du monde par l'intermédiaire d'images, de symboles, de signes, de formes, de représentations, qui assurent aux sociétés, à l'individu, au sujet, une médiation fondamentale avec les lieux, l'espace dans sa complexité. En géographie, notamment, le travail sur l'imagination s'appuie sur une analyse principalement phénoménologique de l'espace » (Dupuy, Puyo, 2015).

De même, elle peut être associée à une poésie des lieux chez Eric Dardel :

"L'espace géographique est unique; il a un nom propre: Paris, Champagne, Sahara, Méditerranée. (...) Le langage du géographe sans effort devient celui du poète» (Dardel, 1990, p. 2-3).

Et qu'y a-t-il de plus «spirituel » que la poésie ? «Le spirituel » est perceptible aussi dans certains travaux comme ceux d'André Siegfried par exemple, à travers ses notions de personnalité ou de tempérament régionaux (Siegfried, 2016). Par conséquent, on sent bien que « le spirituel » est toujours tacite dans l'étude des représentations : la source et l'eau sont souvent symbole de purification et de rédemption spirituelle; les montagnes et les déserts représentent des lieux où l'on se retire en exercices spirituels, en ascèse où objet naturel et esprit deviennent liés; les sites naturels des monastères rappellent que les sociétés, même celles des religieux, étaient paysannes (liens nature/ expressions religieuses) ; grottes et ermitages sont également associés ; de même mers et chapelles et ex-voto pour les disparus; l'habitude aussi de sanctifier des lieux physiques en y fixant des croix; on parle de lieux saints; etc... Les exemples sont légion.

21 «Le spirituel » peut aussi, tout simplement être associée à la beauté : celle qui s'impose à l'œil de l'observateur d'un paysage, et l'invite à une forme ou une autre de contemplation, de méditation, de célébration de la nature en tout cas, et peut-être de prières? La seule contemplation de la nature peut conduire à un sentiment spirituel 
(et/ou religieux) : perception d'un lien qui nous relie au cosmos (bouddhisme -Bareau, 1976-) ? Etat de Grâce (christianisme) ?

«Les religions sont toutes, quelque part, même les plus spiritualistes, des hymnes à la beauté de la nature et à l'harmonie présumée de la Création "(Calvet, Lemartinel, 2002).

Enfin, la dimension spirituelle peut être particulièrement explicite lorsque l'environnement devient une sorte d'espace sacralisé et entraîne un respect quasireligieux de l'intégrité de la nature. En effet, la notion de «spirituel » est présente car en soutenant une transition écologique, et même une conversion écologique, la démarche repose alors sur une base spirituelle fondée sur une conscience des blessures faites à la nature et une éthique environnementale. Dès lors, la personne humaine et la nature, sont mises au cœur du projet de société, dans lequel l'épanouissement humain est (théoriquement) une fin en soi du fait même de cette spiritualité écologique en quelque sorte. Enfin, la référence au «spirituel» peut être encore plus directe lorsqu'elle est portée par les préservationnistes pour qui nature et spiritualité sont consubstantiels de fait :

«La préservation radicale adhère au concept territorial du sanctuaire (Wilderness sanctuary). (...) Cette wilderness est pensée comme une propriété privée pour quelques initiés, "des élus" pouvant communier avec une nature devant rester intouchable pour le commun des mortels. En ce sens les préservationnistes adhèrent à l'idée des parcs naturels à condition qu'ils soient sanctuarisés et réservés à quelques spécialistes. John Muir est un des premiers représentants du préservationnisme radical. C'est un scientifique naturaliste. Il milite en faveur de la préservation des ressources pour leurs valeurs spirituelles et élévatrices» (Tétard, 2011, p. 114-116).

Cette sacralisation absolue de la nature devient une sorte de chemin spirituel radical dont le but est de répondre à la question de la finalité. Protection de la nature et retour des religions sont d'ailleurs concomitants et l'affaiblissement des religions traditionnelles ${ }^{11}$ semblent parfois compensé par cette nouvelle religion : la Nature ellemême. En effet, dans le contexte particulier de notre société christianisée/ déchristianisée où être « sans religion » devient la norme ${ }^{12}$, on peut en réalité identifier deux voies spirituelles selon qu'on inclut Dieu ou pas dans l'explication du monde : soit il s'agit d'une spiritualité horizontale (homme/nature) dans laquelle sans Dieu, l'homme est lui-même le «Créateur » et sacralise, voire sanctifie, la nature ; soit il s'agit d'une spiritualité verticale (Dieu/homme/nature) dans laquelle l'homme est simplement le co-créateur (du fait de la confiance que lui accorde le «Créateur»); Dieu lui ayant abandonné la simple gestion du vivant en quelque sorte. Ces spiritualités constituent deux voies bien distinctes selon qu'on inclut Dieu ou pas pour répondre à la question du sens; « le Spirituel » devenant ainsi, de fait, opératoire. Sans entrer dans une analyse qui nous ferait déborder du sujet et interroger la question sous l'angle exclusivement religieux, on précisera simplement ici, que, d'une part, de tout temps l'humain a sacralisé certains lieux, fleuves, rivières, grottes, montagnes et autre site naturel, et que ces lieux demeurent dans bien des cas encore des lieux de pratiques religieuses qui ont fixé des habitats et des aménagements (sanctuaires, cimetières, églises, chapelles, lavoirs, presbytères...), et qui ont permis le cas échéant, le développement de pèlerinages ${ }^{13}$ (Yvette Veyret le rappelle fort opportunément lors de la table ronde du colloque de 1999 à Cerisy ${ }^{14}$ ) ; et que, d'autre part, dans ce contexte où l'expérience du sacré remonte à la nuit des temps (faisant de l'homme, pour certain, un " homo-religiosus " (Ries, 2009)) se développe actuellement une "théologie écologique ${ }^{15}$ (Pape François, 2015) chez les Chrétiens notamment. «La protection et le respect de l'environnement» sont d'ailleurs passés dans les sondages devant «L'accueil et 
l'intégration des immigrés» (traditionnellement cités avant par les catholiques interrogés, pratiquants ou non, comme valeurs chrétiennes ayant un rôle à jouer dans la société ; par rapport à «L'ensemble des Français » qui les classent après (Fourquet, Pratviel, 2015) $\left.{ }^{16}\right)$ :

"L'église procure donc une nouvelle citoyenneté qui, tout en renversant toutes les frontières sociales, ne se substitue pas mais se superpose à la citoyenneté que le monde nous accorde » (Racine, 1993).

L'ensemble de ces réflexions se présente comme autant d'éléments fragmentaires à approfondir évidemment mais dont nous pouvons d'ores et déjà extraire certains enseignements. Le premier est qu'en regard des éléments exposés, il n'y a aucune raison valable pour mettre à part de la connaissance dans les sciences humaines et sociales cette catégorie du «spirituel » puisque toutes postulent que leur objet d'étude est la personne humaine ${ }^{17}$ dans toutes ses composantes et ses modalités. Par conséquent, le «spirituel » entrant dans cette constitution, il ne doit plus être occulté car aucun humain ne vit hors-spiritualité : "On ne peut pas se passer de communion, de fidélité, d'amour, mais pas non plus de spiritualité » (Comte-Sponville, 2003); celle-ci est une caractéristique intrinsèque, une fonction vivante de chaque être humain (qui le distingue de la bête en quelque sorte) : «En fait, la spiritualité n'est pas une question mais une expérience " (Comte-Sponville, 2003). Il en découle en deuxième lieu que expérience ne peut pas se réduire à une religion, à une sagesse ou à une philosophie particulière, encore moins à une idéologie; «le spirituel» dépasse toutes les organisations sociales. En effet, c'est par l'expérience seule que nous sommes mis en contact avec les réalités; une conception purement idéelle ne saurait finalement fournir l'explication d'aucun faits. Ainsi, la spiritualité comme disposition proprement humaine (ou « le spirituel » pensé à la fois comme expérience humaine mais également ici comme concept) s'exprime à travers toutes les actions et les créations, et dans les relations (sociales et spatiales) et toutes les intellections d'une manière générale. De sorte que, même implicitement, la dimension spirituelle est partout où l'homme est présent et agissant : «Une partie de la vie spirituelle consiste d'abord à habiter ce mystère-là : le mystère de l'être » (Comte-Sponville, 2003). On peut donc légitimement se demander si elle permet l'identification de formations sociales, et spatiales (communautés, et territoires) ? Si « le spirituel» ou la spiritualité sont, comme la religiosité (Bertrand, 1997), facteurs de production spatiale (aménagements d'espaces, sanctuarisation de lieux...) ? S'ils ont le même statut que d'autres processus de construction et de transformation du paysage et de la nature (jusques et y compris dans la symbolique et le sens donné à cette "Nature ») ? D'où, on le voit, il subsiste bien des questions et il y a matière à engager, dans la communauté scientifique universitaire, des travaux sur «le spirituel » comme réalité transversale aux sciences humaines et sociales, en lien avec les milieux et les sociétés, dans le but d'appréhender une vision décomplexée, plus plénière et donc plus ajustée, en tout cas moins bancale, de l'humain :

«Mais qu'il faille désormais construire le discours géographique en regardant et observant une Terre à la fois infiniment rétrécie, et d'une complexité devenue affolante, admettre la certitude (s'il en subsiste une... c'est bien la seule!) que cette complexité est irréductible à l'intelligence humaine, tout cela les géographes mettent quelque temps à le réaliser. » (Chamussy, 2003).

Décembre 2020 


\section{BIBLIOGRAPHIE}

ANDRE Y. (1998), Enseigner les représentations spatiales, Paris, Anthropos-Economica, 254p.

BAILLY A-S. (1977), La perception de l'espace urbain, Paris, Centre de recherche d'urbanisme, 264p.

BAILLY A-S., BEGUIN H. (1990), Introduction à la géographie humaine, Paris, Masson, Coll.

«Géographie », 192p.

BAILLY A-S. (1991), Les concepts de la géographie humaine, Paris, Masson, 247p.

BAILLY A-S., BAUMONT C., HURIOT J-M., SALLEZ A. (1995), Représenter la ville, Paris, Economica, Coll. « Géo. Poche », 192p.

BAREAU A. (1976), « Le Bouddhisme à Ceylan et dans l'Asie du Sud-Est », pp.330-352, In Encyclopédie de la Pléiade Tome.3, Histoire des Religions, Dir. Henri-Charles Puech, Gallimard, 1460p.

BAREL Y. (1986), Espaces, jeux et enjeux, Dir. F. Ariac et R. Brunet, Paris, Fayard, Fondation Diderot, $343 p$.

BARON C. (2011), Littérature et géographie : lieux, espaces, paysages et écritures, Dossier, LHT Le partage des disciplines ; publié le 16 mai 2011, http://www.fabula.org/lht/8/index.php?id=221.

BERTRAND J-R. (1997), « Eléments pour une géographie de la religiosité », Rennes, Norois, PUR, no.174, 416p.

BESSE J-M. (2004), « Le post-modernisme et la géographie. Eléments pour un débat », L'espace Géographique vol.33, no.1/2004, pp.1-5 ; www.cairn.info/revue-espace-geographique-2004-1page-1.htm.

BRUNET R. (1995), Les mots de la Géographie, Dictionnaire critique, 3ème Edition, Paris, Reclus/La Documentation française, Coll. « Dynamiques du Territoire », 518p.

CALVET M., LEMARTINEL B. (2002), « Des religions de la nature, à la nature dans les religions », Intervention $13^{\text {ème }} \mathrm{FIG}$, Géographie et religions, ces croyances, représentations et valeurs qui modèlent le monde, 2002. http://www.fig.saint-die-des-vosges.fr/retro/fig-precedents

CARO M. (2019), « Éprouver l'attachement au lieu : l'épreuve d'un conflit de proximité », L'Espace Politique, Revue en ligne de géographie politique et de géopolitique, no.2019-2, « Rapports à l'espace et formes d'engagement : ancrage, attachement, territorialisation »+Varia, 19p. http:// journals.openedition.org/espacepolitique/6696;DOI:https://doi.org/10.4000/espacepolitique. 6696

CHAMUSSY H. (2003), « Les géographes au risque de la complexité », Géocarrefour, vol.78, no. 1/2003, pp. 61-70.

CHEVALIER D., LEFORT I. (2016), « Le touriste, l'émotion et la mémoire douloureuse », Carnets de géographes, no.9-2016, « Géographie des émotions », Dir. Guinard, Pauline, Tratnjek, Bénédicte. https://doi.org/10.4000/cdg.480

CHEVRIER M-H. (2013), « Pèlerinage et pratiques spatiales à Mexico et à Lourdes : entre familiarité et altérité », Carnets de géographes, no.6-2013, « Géographie des faits religieux », Dir. Dejean, Frédéric, Endelstein, Lucine. https://doi.org/10.4000/cdg.833

CHIVALLON C. (2008), « L'espace, le réel et l'imaginaire : a-t-on encore besoin de la géographie culturelle? », Annales de géographie, vol.660-661, no.2, pp. 67-89. 
COLIGNON B. (2004), Savoirs vernaculaires, Hypergéo, http://www.hypergeo.eu/spip.php?article298

COMTE-SPONVILLE A. (2003), A-t-on encore besoin d'une religion?, Dir. Alain Houziaux Paris, Les Editions de l'atelier-Les Editions ouvrières, Coll. « Questions de vie », 95p.

DARDEL E. (1990), L’homme et la Terre, nature de la réalité géographique, Paris, CTHS, 199p.

DEBARBIEUX B. (2004), Représentation, Hypergéo, http://www.hypergeo.eu/spip.php?article141.

DEJEAN F. (2013), « De la religion dans les sciences sociales aux sciences sociales dans la religion : l'exemple Chrétien », EchoGéo, vol.26-2013, no.01 December 2013. http:// journals.openedition.org/echogeo/13618,https://doi.org/10.4000/echogeo.13618

DESCOLA P., INGOLD T., LUSSAULT M. (2014), Etre au monde, quelle expérience commune ?, Lyon, PUL, Coll. « Grands débats : mode d'emploi », 75p.

DI MEO G. (1991), L’Homme la société l'espace, Paris, Anthropos, 319p.

DI MEO G. (1996), Les territoires du quotidien, Paris, L'harmattan, 207p.

DI MEO G. (1999), Logiques de l'espace, esprit des lieux, Actes du colloque de Géographie de Cerizy-laSalle, Dir. Jacques Lévy et de Michel Lussault, Paris, Belin, Coll. « Mappemonde », 351p.

DUPUY L., PUYO J-Y. (2015), De l'imaginaire géographique aux géographies de l'imaginaire, Ecritures de l'espace, Puppa, Coll. « Spatialités », 176p.

ELISSALDE B. (2004), « Territoire », Hypergéo, http://www.hypergeo.eu/spip.php?article208

FEILDEL B. (2010), Espaces et projets à l'épreuve des affects : pour une reconnaissance du rapport affectif à l'espace dans les pratiques d'aménagement et d'urbanisme, Thèse de Doctorat, Université $\mathrm{F}$. Rabelais de Tours, 651p. HAL Id: tel-00537920 https://tel.archives-ouvertes.fr/tel-00537920Submitted on 19 Nov 2010

FOURQUET J., PRATVIEL E. (2015), « Les domaines dans lesquelles les valeurs chrétiennes ont un rôle positif à jouer ?» Ifop pour Pèlerin Magazine, Département Opinion et Stratégies d'Entreprise, Qui sont les catholiques aujourd'hui ?, Mars 2015, 23 pages. file:///C:/Users/ PLOUCH 1/AppData/Local/Temp/2989-1-study_file-1.pdf

FREMONT A. (1976), La région, espace vécu, Paris, Flammarion, 287p.

FREMONT A. (1998), « Les territoires des hommes », Projet, no.254 Juin, La dynamique des territoires, Revue trimestrielle du CERAS, Paris, Assas Editions VPC, pp.33-38.

GENTELLE P. (Dir.) (2008), Géopolitique du monde contemporain, Paris, Nathan, 288p.

GOULD P., WHITE R. (1984), Cartes mentales, Fribourg (Suisse), Editions Universitaires, traduction Anne Perroud et Michel Roten, 188p.

Grand Corps Malade (2006), Midi 20, Anouche Production Universal Music.

HERIN R. (1986), « Une Géographie des rapports sociaux », L'espace géographique, no.2, Paris, Doin, pp108-110.

KELEN J. (2015), Bréviaire du colimaçon; sur la vie spirituelle, Paris, DDB Poche, 153p.

LAFONT P-B. (1976), pp.367, « Le Bouddhisme vietnamien », pp.353 à 370, In Encyclopédie de la Pléiade Tome3, Histoire des Religions, Dir. Henri-Charles Puech, Paris, Gallimard, 1460p.

LEFORT I. (2005), Géopolitique du développement durable, Paris, PUF, 384p.

LEVY J., LUSSAULT M. (Dir.), (2000), Logiques de l'espace, esprit des lieux. Géographies à Cerisy, Actes du colloque de 1999, Paris, Belin, 351p. 
LYNCH K. (1999), L'image de la Cité, trad. par Marie-Françoise et Jean-Louis Vénard de The Image of the City (1960), Paris, Dunod, 221p.

NIEL X. (1998), «L'état de la pratique religieuse en France », Insee-4 pages vol.570 - no.Mars 1998Division Conditions de vie des ménages, $4 \mathrm{p}$.

Pape François, (2015), Lettre encyclique Laudato Si' sur la sauvegarde de la maison commune, donnée à Rome pour la Pentecôte, le 24 mai 2015, http://w2.vatican.va/content/francesco/fr/encyclicals/ documents/papa-francesco_20150524_enciclica-laudato-si.html

PAULET J-P. (2002), Les représentations mentales en géographie, Paris, Economica, 152p.

PECH M-E. (2013), « La déchristianisation touche de plus en plus de jeunes Français », Le Figaro Société, publié le 29/03/2013, mis à jour le 29/03/2013 https://www.lefigaro.fr/actualite-france/ 2013/03/29/01016-20130329ARTFIG00421-la-dechristianisation-touche-de-plus-en-plus-dejeunes-francais.php

PINCHEMEL P. et G. (1997), La face de la terre-Eléments de géographie, vol.IV, Paris, Armand Colin, $382 \mathrm{p}$.

PLOUCHART L. (1997), De la cité au quartier, le grand ensemble et ses représentations. Agglomérations d'Angers et Le Mans, Thèse de Doctorat de Géographie, Université du Maine, Le Mans, Dirigée par le Professeur Jean-René Bertrand, 268p+livret d'annexes.

PLOUCHART L. (1999), Comprendre les grands ensembles, une exploration des représentations et des perceptions, Paris, L'harmattan, Coll. « Villes \& Entreprises », 296p.

PLOUCHART L., SECHET R., GARAT P. (2000), Contrat Local de Sécurité de l'agglomération redonnaise, rapport d'étude au CCAS de Redon, 50p.

RACINE J-B. (1993), La ville entre Dieu et les hommes, Paris, Anthropos-Economica, 354p.

RACINE J. (1984), « Pour une géographie combinatoire », L'espace géographique, no.4 Oct./Déc., Paris, CNRS, Doin Editeurs, pp317-328.

REGNIER-LOILIER A., PRIOUX F. (2008), « La pratique religieuse influence-t-elle les comportements familiaux? », Population \& Sociétés, vol.447, no.Juillet-août 2008, Bulletin mensuel d'information de l'INED, $4 \mathrm{p}$.

RIES J. (2009), L'homo-religiosus et son expérience du sacré. Introduction à une nouvelle anthropologie religieuse, Paris, Cerf, Coll. « Patrimoines-Histoire des religions », 524p.

ROUGERIE G. (1975), Les cadres de vie, Paris, PUF, Coll. « SUP Le géographe », 264p.

SIEGFRIED A. (2016), Le tableau politique de la France de l'Ouest. 100 ans après, héritages et postérités, Rennes, PUR, Coll. « Géographie sociale », 350p.

TETARD F. (2011), Géographie des conflits, Paris, Sedes, 342p.

VAUCHEZ A. (1994), La spiritualité au Moyen-Age occidental $8^{\text {ème-1}} 13^{\text {ème }}$ siècle, Paris, Seuil, Coll. «Point Histoire $", 212 p$.

VERGNOLLE-MAINAR C., DESAILLY B. (2005), Territoires, risques, développement, éducation, Paris, SCEREN, CRDP Midi-Pyrénées, 364p.

VEYRET Y. (1999), Nature, Géographie, Environnement, Les actes du FIG, http://archives-fig-stdie.cndp.fr/actes/actes_2002/veyret/article.htm 


\section{NOTES}

1. C'est-à-dire celui qu'en donnerait tout bonnement "Le Petit Larousse illustré »: "Qualité de ce qui est esprit, de ce qui est dégagé de toute matérialité : La spiritualité de l'âme, de la poésie. Ce qui concerne la doctrine ou la vie centrée sur Dieu et les choses spirituelles ».

2. Colloque international et interdisciplinaire Rennes2, 19 et 20 octobre 2017, Le spirituel, un concept opératoire en sciences humaines ? porté par "Theorias " Réseau transdisciplinaire et international de théorisation de la spiritualité direction scientifique Claude Le Fustec (France), Myriam Watthée-Delmotte (Belgique), Xavier Gravend-Tirole (Suisse). Une publication est actuellement à l'étude.

3. "L'espace géographique qui accueille la vie sociale et au sein duquel s'accomplissent les destinées humaines, n'a pas cette objectivité placide que la géographie classique et ses versions positivistes retenaient naguère implicitement. (...) L'homme qui se représente l'espace et son milieu, qui le façonne et le transforme est à la fois une conscience, une psyché, un sujet philosophique et politique, un individu, une personne, un être humain et un être social » (Di Méo, 1999).

4. Ici un exemple tiré de l'appel à communication de la revue Territoire en mouvement en 2018, «Du visible et de l'invisible dans la fabrique de la Ville et les études urbaines» : «(...) En s'emparant explicitement de cette dialectique du visible ou de l'invisible comme catégorie d'analyse (par exemple: Bonnin, 2008; Choné, 2012 ; De Boeck et Plissart, 2005 ; Devisme, 2014 ; Latour et Hermant, 2009), ou plus souvent implicitement, en mobilisant alors d'autres dialectiques: inclusion/exclusion; centre/ périphérie ; dominant/dominé; matériel/immatériel ; formel/représentations; visuel/multisensoriel ... ».

5. Pour les géographes le concept de DD est d'autant plus difficile qu'il fait passer de l'espace, de la spatialisation, à la catégorie du temps futur pour les sociétés : «Pendant plus de 50 ans, les géographes vidaliens en France n'ont fait qu'exprimer une "durabilité » des milieux, qu'on appelait alors permanence » (Lefort, 2005, p.63-65).

6. "(...) Il ne serait guère pertinent, dans la prise en compte des représentations des sociétés étudiées, que le chercheur omette de tenir compte, pour les contrôler autant qu'il se peut, de ses propres représentations. » (Racine, 1984).

7. «La majorité des travaux géographiques considèrent aujourd'hui que les facteurs essentiels sont à chercher dans les actions multiples des sociétés humaines. Cela exclut naturellement de commencer l'analyse géographique d'un espace par l'étude des données physiques, du cadre dit naturel qui, en fait, n'est plus le moteur, le déterminant des évolutions sociales qu'il a pu être dans certaines situations anciennes» (Veyret, 1999, non paginé). Dans un article, le géographe Armand Frémont pose quant à lui tout bonnement la question de savoir si les territoires ont encore du sens : "L'homme contemporain a définitivement rompu l'équilibre entre enracinement et mouvement. Depuis le Néolithique c'est la plus grande révolution survenue dans la géographie humaine » (Frémont, 1998, p.33-38).

8. Colloque de géographie Cerisy-la-Salle, Juin 2018, Saisir le rapport affectif aux lieux; CITERESUMR7324; www.ccic-cerisy.asso.fr.

9. Sonores, olfactives, tactiles, visuelles (ou même plus ou moins conscientes et objectives, on pense au sentiment d'insécurité (qui n'est pas l'insécurité) (Plouchart, Séchet, Garat, 2000)).

10. "C'est ainsi que l'on est arrivé à la définition du Conseil International de la Langue Française où l'environnement constitue "L'ensemble à un moment donné, des agents physiques, chimiques, biologiques et des facteurs sociaux susceptibles d'avoir un effet direct ou indirect, immédiat ou à terme, sur les êtres vivants et les activités humaines » (Vergnolle-Mainar, Desailly, 2005, p.6-7). 
11. "En 2005, environ 35 millions des 18-79 ans (80\%) ont ainsi pour religion d'origine ou d'appartenance le catholicisme, un peu plus de 2 millions (5\%) se déclarent musulmans, 900000 (2\%) protestants et 800000 (2\%) d'une autre confession ; 5 millions (11\%) disent n'avoir aucune religion » (Régnier-Loilier, Prioux, 2008).

12. «Les personnes « sans-religion " constituent désormais le groupe le plus important chez les personnes âgées de moins de 35 ans. Leur proportion atteint 47\% chez les jeunes âgés de 18 à 24 ans et décroît progressivement avec l'âge des individus pour s'établir à seulement $16 \%$ chez les plus de 65 ans. » (Pech, 2013).

13. Voir aussi sur les pèlerinages Chevrier, 2013.

14. Dont les actes ont été publiés en 2000 (Lévy, Lussault, 2000).

15. In Laudato Si, «Loué sois-tu » diffusé en mai 2015 sous le titre : «Sur la sauvegarde de la maison commune »).

16. En effet, lorsqu'on pose la question suivante : selon vous, dans quels domaines les valeurs chrétiennes ont-elles un rôle positif à jouer dans la société française d'aujourd'hui ? (2 réponses possibles), les réponses sont :1-La famille et l'éducation ; 2La solidarité envers les plus pauvres ; 3-Le dialogue entre les différentes cultures et les différentes religions; 4-La bioéthique, le respect de la vie; 5-La moralisation du capitalisme ; 6-L'accueil et l'intégration des immigrés ; 7-La protection et le respect de l'environnement ; 8-La construction européenne.

17. « Le groupe humain d'abord, l'espace ensuite... Ce qui est premier en géographie sociale c'est la société » (Hérin, 1986, p.108-110).

\section{RÉSUMÉS}

L'article montre que « Le spirituel » est une notion utilisée dans les sciences humaines et sociales, et en géographie, par le simple fait que l'être humain est corporel et «spirituel ». La question de savoir si « le spirituel » peut être considéré comme un concept scientifique opératoire intéresse toutes les sciences humaines et sociales y compris la géographie qui use de la notion dans les travaux sur les représentations territoriales, les perceptions spatiales et les comportements humains. Cela dit, comme réalité transversale aux sciences humaines et sociales, en lien avec les milieux et les sociétés, c'est sans doute dans les travaux concernant l'environnement (et sa protection) que la dialectique matériel/«spirituel» est la plus saillante; et les liens environnement/spiritualité sont bien manifestés dans le concept de développement durable notamment.

The article shows that "The spiritual" is a notion used in the humanities, social sciences, and geography, by the fact that the human being is corporeal and "spiritual". The question of whether «the spiritual» can be considered as an operational scientific concept is of interest to all the humanities and social sciences including the geography which uses the notion in the work on the territorial representations, spatial perceptions and human behaviour. That said, as a crosscutting reality in the humanities and social sciences, in connection with environments and societies, it is probably in the work concerning the environment (and its protection) that the 
material/"spiritual" dialectic is most prominent; and the links between environment/spirituality are well reflected in the concept of sustainable development in particular.

\section{INDEX}

Thèmes : Carnets de recherches

Mots-clés : Spirituel, spiritualité, Environnement/nature, Images, expériences, représentations et perceptions spatiales, Espaces vécus, perçus et représentés.

Keywords : Spiritual, spirituality, Environment / nature, Images, experiences, representations and spatial perceptions, Spaces lived, perceived and represented.

\section{AUTEUR}

\section{LOUISA EVEN}

Maître de Conférences-Université Rennes2. Laboratoire ESO Rennes-CNRS, ESO - UMR 6590 Espaces et Sociétés. louisa.even[at]univ-rennes2.fr 